\title{
The effect of immunonutrition on bacterial translocation after Pringle maneuverer in rats
}

\author{
Ozkan Subasi ${ }^{1}$, Selim Yigit Yildiz ${ }^{1}$, Adem Yuksel ${ }^{2}$, Murat Coskun ${ }^{1}$, Hamdi Taner Turgut ${ }^{1}$, Eda Yildiz ${ }^{3}$ \\ ${ }^{1}$ Department of General Surgery, Kocaeli Derince Training and Research Hospital, Kocaeli, Turkey \\ 2Department of Gastrointestinal Surgery, Kocaeli Derince Training and Research Hospital, Kocaeli, Turkey \\ ${ }^{3}$ Department of Clinical Microbiology, Kocaeli Derince Training and Research Hospital, Kocaeli, Turkey
}

Gastroenterology Rev 2019; 14 (3): 178-182

DOI: https://doi.org/10.5114/pg.2019.88166

Key words: immunonutrition, Pringle manoeuvre, bacterial translocation.

Address for correspondence: Ozkan Subasi MD, Department of General Surgery, Kocaeli Derince Training and Research Hospital, 41900 Kocaeli, Turkey, phone: +90 26231780 00/+90 50538421 09, fax: +90 262233 46 41, e-mail: osubasi25@hotmail.com

\begin{abstract}
Introduction: Temporary clamping of the hepatoduodenal ligament (the Pringle manoeuvre) is the most commonly used method to prevent intraoperative blood loss in liver surgery. Some side effects of the Pringle manoeuvre (PM) can occur.

Aim: To investigate the effectiveness of preoperative immunonutritional support to prevent bacterial translocation (BT) in rats due to $P M$.

Material and methods: Forty Wistar albino rats were randomly divided into four groups. Groups 1 and 2 were fed with normal rat diet and water, and groups 3 and 4 were fed with enteral immunonutrition (Impact Glutamine) containing $1 \mathrm{~g} / \mathrm{kg} / \mathrm{day}$ amino acid in the preoperative period. Group $1(n=10)$ and $4(n=10)$ rats were treated only with laparotomy; group $2(n=10)$ and $3(n=10)$ rats were treated with PM for 30 min with laparotomy. After 30 min, relaparotomy was applied to all groups and portal blood, mesentery, spleen samples were taken for culture purposes.

Results: Proliferation in portal blood cultures was significantly higher in the samples from the normally fed group (group 2) in whom PM was applied, compared to the other groups $(p<0.001)$. No proliferation was observed in the PM-treated group (group 3), who also received preoperative immunonutritional support.

Conclusions: Preoperative immunonutritional support is effective in the prevention of BT due to PM in rats.
\end{abstract}

\section{Introduction}

In liver surgery, the most important factors affecting morbidity and mortality are intraoperative blood loss and septic complications [1]. Various techniques have been used to prevent intraoperative blood loss. The oldest and most commonly used of these techniques is the Pringle manoeuvre (PM). The PM was first discovered in 1908 by J.H. Pringle, and it was proven that temporary clamping of the hepatoduodenal ligament reduces intraoperative blood loss [2]. The PM causes portal, superficial, and inferior mesenteric venous stasis, which leads to a decrease in splanchnic blood flow [3]. Experimental studies have shown that splanchnic hypoperfusion secondary to the PM causes intestinal mucosal injury and bacterial translocation (BT) [4-7]. Intestinal mucosal injury and BT have been shown to be predisposing factors in the development of serious clinical conditions after trauma, major surgery, and shock, such as sepsis, and multiorgan failure $[8,9]$.

Immunonutrition is a nutritional product containing high energy and protein, enriched with certain nutrients, mostly arginine, glutamine, omega- 3 fatty acids, and nucleotides. The use of these products 5-7 days prior to various surgical procedures has been shown to reduce postoperative infectious complications [10]. It has also been shown to reduce BT in experimental obstructive jaundice and intestinal obstruction models $[11,12]$.

\section{Aim}

In this study, it was planned to investigate the effects of preoperative immunonutritional support against development of BT due to PM, which is commonly used in liver surgery. 


\section{Material and methods}

The study protocol was made with the approval of Kocaeli University Experimental Animal Ethics Committee. Forty Wistar albino rats weighing 200-250 g were used in the study. Rats were housed in a 12-hour light/ dark cycle at constant temperature in accordance with the standards of the National Institutes of Health Publication Guide for the Care and Use of Laboratory Animals (NIHPGC). Rats were randomly sampled and divided into four groups with 10 rats in each group (Table I). All groups were fed for 5 days according to their characteristics. Groups 1 and 2 were fed with standard rat diet and water, and groups 3 and 4 were supplemented with $1 \mathrm{~g} / \mathrm{kg} /$ day amino acid containing enteral nutritional supplement (22\% protein, $51 \%$ carbohydrate, $24 \%$ fat, also containing L-arginine $14 \mathrm{~g} / \mathrm{l}$, L-glutamine $10 \mathrm{~g} / \mathrm{l}$ ) and fed with a paediatric orogastric feeding probe in addition to standard rat diet and water.

\section{Surgical procedure}

All subjects, kept under standard laboratory conditions, were allowed to drink water while being fasted on the day of operation at the end of the fifth day. Anaesthesia was induced by administering intramuscular $100 \mathrm{mg} / \mathrm{kg}$ ketamine hydrochloride and $10 \mathrm{mg} / \mathrm{kg}$ xylazine hydrochloride combination to all subjects in the group.

After anaesthesia, the anterior abdominal wall was cleansed with povidone-iodine and then the hepatoduodenal ligament was released by exploration of the abdomen through a $2 \mathrm{~cm}$ upper abdominal incision, as standard. The hepatoduodenal ligament was occluded for 30 min in the subjects of groups 2 and 3 using a vascular clamp. At the end of $30 \mathrm{~min}$, reperfusion was provided, and the sac was closed with 3/0 Vicryl. The hepatoduodenal ligament was explored with laparotomy in group 3 and 4 subjects. After 30 min of waiting, the abdomen was closed with $3 / 0$ Vicryl. All subjects in the groups underwent relaparotomy $30 \mathrm{~min}$ after the closure of the abdomen. The portal vein was cannulated with a gauge catheter and a 2-ml blood sample was taken. Samples from the spleen and terminal ileum were taken for microbiological examination. All surgical procedures were performed using sterile instruments in aseptic conditions. After the procedure, the rats were sacrificed by cardiac incision.

\section{Microbiological analysis}

Microbiological analyses of the study were carried out in Kocaeli Derince Training and Research Hospital Microbiology Laboratory. During microbiological analysis, tissue samples from the meso of small intestine were placed in sterile tubes containing $2 \mathrm{ml}$ Brain Heart Infusion Broth (RTA Laboratories, Turkey). After homogenisation, direct seeding was carried out for qualitative evaluation and for quantitative evaluation, $100 \mu \mathrm{l}$ was inoculated into 5\% Sheep Blood Agar (RTA Laboratories, Turkey) and Eosine Metilen Blue Agar (RTA Laboratories, Turkey), and then the plates were incubated for $24 \mathrm{~h}$ at $37^{\circ} \mathrm{C}$. Incubation of plates without reproduction was extended to $48 \mathrm{~h}$. Tissue samples taken from the spleen were collected into sterile tubes with 2 ml Brain Heart Infusion Broth (RTA Laboratories, Turkey). After homogenisation, direct seeding was carried out for qualitative evaluation and for quantitative evaluation, $100 \mu \mathrm{l}$ was inoculated into 5\% Sheep Blood Agar (RTA Laboratories, Turkey) and Eosine Metilen Blue Agar (RTA Laboratories, Turkey), and then the plates were incubated for $24 \mathrm{~h}$ at $37^{\circ} \mathrm{C}$. Incubation of plates without reproduction was extended to $48 \mathrm{~h}$. Two-millilitre blood samples taken from the portal vein were incubated in a BacT/ALERT ${ }^{\oplus}$ 3D (bioMERIEUX, France) automated blood culture device after being inoculated into BacT/ALERT ${ }^{\oplus} 3 \mathrm{D}$ (bioMERIEUX, France) blood culture bottles.

Reproducible microorganisms were identified by a VITEK ${ }^{\oplus} 2$ Compact (bioMERIEUX, France) automated identification and antibiogram instrument.

\section{Statistical analysis}

Statistical evaluation was performed with IBM SPSS 20.0 (SPSS Inc., Chicago, IL, USA) package programme. Categorical variables were given as frequencies (percentages). Differences between groups were assessed by Monte Carlo $\chi^{2}$ analysis. $P<0.05$ was considered sufficient for statistical significance in two-way tests.

Table I. Groups $(n=10)$

\begin{tabular}{lrr} 
Group & Preoperative feeding (5 day) & Operation \\
\hline 1 & Standard rat diet & Laparotomy \\
\hline 2 & Standard rat diet & Laparotomy + PM \\
\hline 3 & Standard rat diet + enteral nutritional supplement & Laparotomy + PM
\end{tabular}

PM - Pringle manoeuvre. 
Table II. Distribution of microorganism species isolated from blood cultures

\begin{tabular}{lcccc} 
Microorganism species & Group 1 & Group 2 & Group 3 & Group 4 \\
\hline Enterococcus faecalis & 0 & $2(20 \%)$ & 0 & 0 \\
\hline Leuconostoc pseudomesenteroides & 0 & $1(10 \%)$ & 0 & 0
\end{tabular}

\section{Results}

During the experiment, all subjects tolerated the intervention, and no subjects were lost within the estimated period or left out of the study. Growth in blood cultures were detected in three samples (30\%) of group 2 (standard feed + PM), while no growth was detected in the other groups. In the statistical analysis of all groups, the proliferation in blood cultures of group 2 was statistically higher than in the other groups $(p<0.001)$ (Table II). All of the proliferation in blood cultures occurred within the first $48 \mathrm{~h}$ after inoculation. Enterococcus faecalis growth was seen in two samples of portal vein culture of group 2 and Leuconostoc pseudomesenteroides growth was seen in one sample (Figure 1).

Growth in mesenteric lymph node specimens was $10 \%$ in group 2, and no growth was observed in other groups (Figure 2). When compared with other groups, there was no statistically significant difference, but there was a proliferation in group $2(p=1.00)$. Enterococcus faecalis had proliferated in the mesenteric lymph node samples of a rat from group 2. No spleen samples were found to have any growth; no statistical comparison was made for this reason.

\section{Discussion}

BT is defined as the passage of microorganisms or their products (endotoxin), which are localised in the intestinal organs to extraintestinal areas that are normally sterile, such as mesenteric lymph nodes, other intestinal organs, and bloodstream, by crossing the intes-

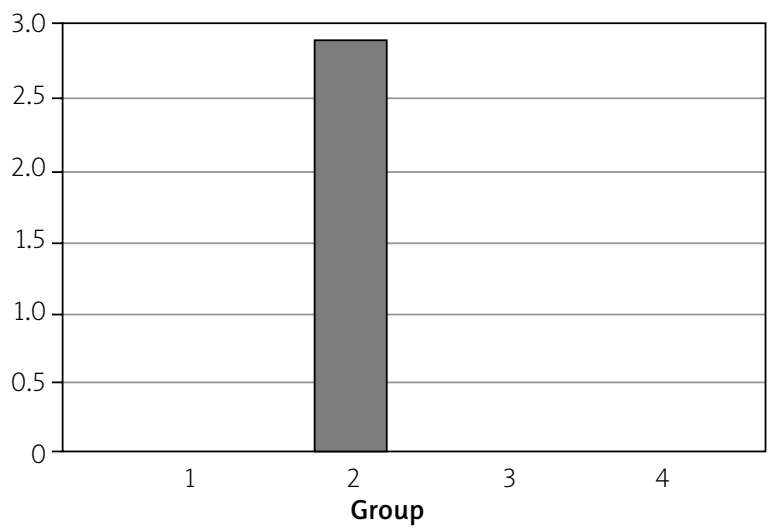

Figure 1. Blood culture tinal mucosal barrier [13]. There are different opinions about the clinical importance of BT. There are opinions suggesting that BT can be considered as a physiological event in normal healthy individuals [14]. However, contrary to this view, O'Boyle et al. [15] conducted a study in which BT was found in $15 \%$ of patients undergoing laparotomy, and $45 \%$ of the patients with BT had postoperative sepsis, which was reported to be $19 \%$ in the group without translocation. In addition, other studies supporting this view have reported that BT is a predisposing factor in the development of postoperative infectious complications in patients undergoing major abdominal surgery $[16,17]$.

PM, which is commonly used for intraoperative bleeding control in liver surgeries, has been shown to lead BT in mesenteric lymph nodes and portal blood specimens in various experimental and clinical studies [18-21]. Our study also showed that PM causes bacterial growth in portal blood and mesenteric tissue cultures in rats. Although there is sufficient information in the literature about the clinical conditions that cause BT, information about the anatomic pathway (lymphatic-hematogenous) that is effective in BT development and when it first developed is insufficient [22]. In our study, it was observed that there was growth in more rats in the portal blood cultures taken at the first hour after PM application, compared to the mesenteric culture samples. In a study done to explain the anatomic pathway in BT, it was shown that portal blood cultures in the early period had a higher rate of growth than mesenteric cultures, and the hematogenous pathway was the main pathway of BT [23].

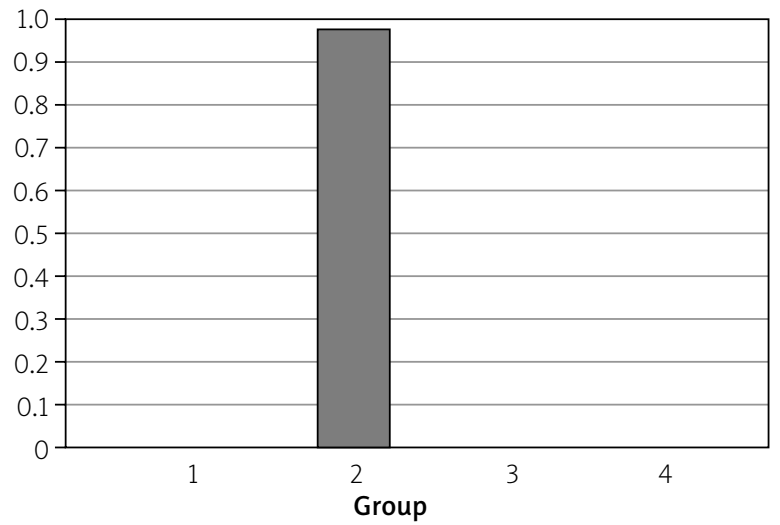

Figure 2. Mesenteric lymph node culture 
There are few experimental studies to prevent BT after PM. In these few experimental studies, it has been shown that the use of prophylactic antibiotics, preoperative bowel cleansing, and the use of low-dose dexamethasone prevent BT after PM [18, 24].

It has been reported that the use of enteral immunonutrition products containing glutamine, arginine, nucleotide, and omega-3 fatty acids in different combinations and doses prior to surgery regulates the inflammatory response in the early period and strengthens the immune response and bowel function [25]. Glutamine has been shown to inhibit intestinal atrophy by increasing epithelial cell proliferation, to decrease bacterial adhesion to the intestinal mucosal surface by increasing levels of secretory immunoglobulin $A$ and mucin, and to prevent BT by helping to preserve the integrity of the intestinal wall [26]. In a study conducted by Zou et al. in a severe acute pancreatitis model, enteral immunonutrition was reported to enhance the intestinal mucosa barrier system and the immune system [27]. In an obstructive jaundice model, the use of enteral immunonutrition products in preoperative and postoperative periods has been shown to reduce BT and intestinal villus atrophy [11]. Although histopathological examination was not included in our study, preoperative immunonutrition support was shown to prevent BT after PM, according to microbiologic studies.

\section{Conclusions}

In this study, based on microbiological findings, it was shown that PM leads to BT, and preoperative immunonutrition support prevents BT due to PM. However, uncertainties remain about how much $\mathrm{BT}$ is responsible for the development of postoperative septic complications. In light of our study and previous studies, the effect of immunonutritional support on BT and infectious complications in patient groups who undergo a surgical technique with a high risk of BT, such as PM, and other groups with risk of BT (burn, trauma, pancreatitis, cirrhosis) should be investigated in future clinical trials.

\section{Conflict of interest}

The authors declare no conflict of interest.

\section{References}

1. Katz SC, Shia J, Liau KH, et al. Operative blood loss independently predicts recurrence and survival after resection of hepatocellular carcinoma. Ann Surg 2009; 249: 617-23.

2. Pringle JH. V. Notes on the arrest of hepatic hemorrhage due to trauma. Ann Surg 1908; 48: 541-9.

3. Belghiti J, Marty J, Farges O. Techniques, hemodynamic monitoring, and indications for vascular clamping during liver resections. J Hepatobiliary Pancreat Surg 1998; 5: 69-76.
4. Ochiai H, Nakamura S, Suzuki S, Baba S. Pancreatic damage resulting from temporary portal triad interruption during partial hepatectomy: protective effect of a prostaglandin 12 analogue. J Surg Res 1997; 73: 129-36.

5. Filos KS, Kirkilesis I, Spiliopoulou I, et al. Bacterial translocation, endotoxaemia and apoptosis following Pringle manoeuvre in rats. Injury 2004; 35: 35-43.

6. Unno N, Uchiyama T, Yamamoto N, et al. Portal triad occlusion induces endotoxin tolerance: role of portal congestion. J Surg Res 2006; 135: 213-7.

7. Dello SA, Reisinger KW, van Dam RM, et al. Total intermittent Pringle maneuver during liver resection can induce intestinal epithelial cell damage and endotoxemia. PLoS One 2012; 7 : e30539.

8. Derikx JP, Poeze M, van Bijnen AA, et al. Evidence for intestinal and liver epithelial cell injury in the early phase of sepsis. Shock (Augusta, Ga) 2007; 28: 544-8.

9. Holland J, Carey M, Hughes N, et al. Intraoperative splanchnic hypoperfusion, increased intestinal permeability, down-regulation of monocyte class II major histocompatibility complex expression, exaggerated acute phase response, and sepsis. Am J Surg 2005; 190: 393-400.

10. Mariette C. Immunonutrition. J Visceral Surg 2015; 152 Suppl 1: S14-7.

11. Zulfikaroglu B, Zulfikaroglu E, Ozmen MM, et al. The effect of immunonutrition on bacterial translocation, and intestinal villus atrophy in experimental obstructive jaundice. Clin Nutr 2003; 22: 277-81.

12. Quirino IE, Correia MI, Cardoso VN. The impact of arginine on bacterial translocation in an intestinal obstruction model in rats. Clin Nutr 2007; 26: 335-40.

13. Berg RD. Translocation of enteric bacteria in health and disease. Curr Stud Hematol Blood Transfus 1992; 59: 44-65.

14. Sedman PC, Macfie J, Sagar P, et al. The prevalence of gut translocation in humans. Gastroenterology 1994; 107: 643-9.

15. O'Boyle CJ, MacFie J, Mitchell CJ, et al. Microbiology of bacterial translocation in humans. Gut 1998; 42: 29-35.

16. Deitch EA. Gut-origin sepsis: evolution of a concept. Surgeon 2012; 10: 350-6.

17. MacFie J, O’Boyle C, Mitchell CJ, et al. Gut origin of sepsis: a prospective study investigating associations between bacterial translocation, gastric microflora, and septic morbidity. Gut 1999; 45: 223-8.

18. Erenoglu B, Gokturk HS, Kucukkartallar T, et al. Mechanical intestinal cleansing and antibiotic prophylaxis for preventing bacterial translocation during the Pringle maneuver in rabbits. Surg Today 2011; 41: 824-8.

19. Yeh DC, Wu CC, Ho WM, et al. Bacterial translocation after cirrhotic liver resection: a clinical investigation of 181 patients. J Surg Res 2003; 111: 209-14.

20. Ferri M, Gabriel S, Gavelli A, et al. Bacterial translocation during portal clamping for liver resection. A clinical study. Arch Surg 1997; 132: 162-5.

21. Su Y, Pan H, Guo Z, et al. Bacterial translocation and endotoxemia after pringle maneuver in cirrhotic rats. Dig Dis Sci 2015; 60: 414-9.

22. Wiest R, Lawson M, Geuking M. Pathological bacterial translocation in liver cirrhosis. J Hepatol 2014; 60: 197-209. 
23. Mainous MR, Tso P, Berg RD, Deitch EA. Studies of the route, magnitude, and time course of bacterial translocation in a model of systemic inflammation. Arch Surg 1991; 126: 33-7.

24. Karaman K, Tirnaksiz MB, Ulusu N, et al. Effects of dexamethasone on ischemia reperfusion injury following pringle maneuver. Hepatogastroenterology 2011; 58: 465-71.

25. Braga M. Perioperative immunonutrition and gut function. Curr Opin Clin Nutr Metab Care 2012; 15: 485-8.

26. van der Hulst RR, van Kreel BK, von Meyenfeldt MF, et al. Glutamine and the preservation of gut integrity. Lancet 1993; 341: 1363-5.

27. Zou XP, Chen M, Wei W, et al. Effects of enteral immunonutrition on the maintenance of gut barrier function and immune function in pigs with severe acute pancreatitis. J Parenter Enteral Nutr 2010; 34: 554-66.

Received: 3.11 .2018

Accepted: 16.01.2019 